\title{
IDENTIFICAÇÃO DOS INSULINOMAS PELA ECOENDOSCOPIA
}

\author{
José Celso Ardengh*, loana Heuko Valiati, Stephan Geocze \\ Trabalho realizado no Hospital São Paulo e Hospital Israelita Albert Einstein, São Paulo, SP.
}

RESUMO - Objetivo. Demonstrar a sensibilidade da ecoendoscopia (EE) no diagnóstico pré-operatório dos insulinomas e comparar com outros testes diagnósticos como tomografia helicoidal e ressonância magnética.

Métodos. Trinta pacientes foram examinados prospectivamente pela ecoendoscopia com o diagnóstico clínico de insulinoma antes de serem submetidos ao tratamento cirúrgico. 0 exame ecoendoscópico sempre foi precedido pelo US, TC helicoidal e em 10 pacientes pela RM. Em 12 casos foi indicada a punção aspirativa com agulha fina eco-guiada para confirmar a suspeita de insulinoma.

RESULTADOs. A sensibilidade da EE na identificação dessas lesões foi de $86,6 \%$ (26/30 tumores). Vinte e seis tumores foram benignos $(86,6 \%)$ e quatro malignos (13,4\%). Realizamos punção biópsia aspirativa em 12 pacientes (40\%) e o diagnóstico histológico foi feito em I0/12 pacientes (83,3\%). Nessa casuística, 25 tumores foram menores que $2 \mathrm{~cm}(83,3 \%)$ e cinco maiores que $2 \mathrm{~cm}(16,7 \%)$. Os tumores detectados pela EE tinham tamanho médio de $1,5 \mathrm{~cm}(0,6$ a $5,4 \mathrm{~cm}$ ). A identificação dos tumores pela EE na cabeça, corpo e cauda foi de $100 \%, 100 \%$ e $55,5 \%$, respectivamente.

Conclusão. A EE tem alta sensibilidade na identificação e localização dessas lesões e deve ser recomendada quando os métodos tradicionais de imagem empregados falham no diagnóstico. A PAAF é uma tentativa para evitarmos falso-positivos.

Unitermos: Diagnóstico. Insulinoma. Ecoendoscopia. Punção aspirativa com agulha fina.

\section{INTRODUÇÃo}

Os insulinomas são raros e dos tumores secretores pancreáticos os mais comuns'. A hipoglicemia é o sintoma mais freqüente, podendo ocorrer choque insulínico com o jejum, mas com alivio dos sintomas com a infusão de glicose. Geralmente pequenos $(66 \%<2 \mathrm{~cm}$ e $24 \%<1 \mathrm{~cm}$ ), benignos, solitários e intrapancreáticos, localizam-se de forma semelhante em todas as regiões do pâncreas ${ }^{1,2}$.

Como a ressecção cirúrgica constitui o tratamento de escolha e a grande maioria dessas lesões são benignas e solitárias, o diagnóstico preciso pré-operatório é de suma importância. Apesar dos avanços na área da imagem, a localização exata desses tumores ainda é um desafio. As técnicas não-invasivas como o ultra-som abdominal (US) e a tomografia computadorizada helicoidal (TC), seriam inicialmente preferíveis, se não fosse o fato desses tumores serem diminutos, tornando-os de difícil detecção por esses métodos, que apresentam sensibilidade ao redor de $15 \%$ e $64 \%$, respectivamente 3 .

*Correspondêncio

Alameda dos Arapanés, 881 - Ci. 111

CEP: 04524-001 - São Paulo - SP

Tel. (11) 5055-7134 - E-mail: jcelso@uol.com.br
A angiografia (AG) e a cateterização venosa trans-hepática (CVTH) têm maior sensibilidade se comparados aos anteriores, porém são exames de alto custo e de difícil realização, podendo apresentar resultados falso-positivos, falso-negativos e complicações ${ }^{1,4,5}$. O ultra-som intra-operatório (USIO) localiza 90\% deles ${ }^{1,3}$, mas tecnicamente é difícil, além de apresentar como complicação a lesão dos vasos esplênicos devido a mobilização da glândula do retroperitônio.

A ecoendoscopia (EE) fornece imagens de todo o pâncreas com excelente resolução. É possível o estudo do parênquima do processo uncinado, cabeça, corpo e cauda, além de observarmos com precisão sua relação com estruturas vasculares periféricas. $O$ ducto pancreático principal (DPP) pode ser estudado com facilidade, bem como a via biliar extrahepática. Pequenas lesões de 2 a $5 \mathrm{~mm}$ podem ser detectadas. Estudos retrospectivos demonstram que a EE identifica e estadia tumores pancreáticos com sensibilidade que varia de $80 \%$ a $90 \%$ 4,6,7.

Baseado nesses aspectos, este estudo tem como objetivo demonstrar a sensibilidade da EE na localização precisa dos insulinomas e compará-la com outros testes diagnósticos (US, TC helicoidal, RM e USIO) em pacientes com suspeita clínica de insulinoma.

\section{Métodos}

\section{Seleção dos pacientes}

De março de 1993 a setembro de 2002, 6.268 exames de ecoendoscopia foram realizados no setor de Endoscopia Digestiva da disciplina de Gastroenterologia da UNIFESP e no setor de Endoscopia e Ecoendoscopia do Hospital Israelita Albert Einstein, sempre pelo mesmo examinador (J.C.A.).

Trinta e seis pacientes $(0,5 \%)$ com forte suspeita clínica de insulinoma por apresentarem hipoglicemia e níveis séricos elevados de insulina foram encaminhados para a realização do exame ecoendoscópico. Todos os pacientes tinham US, TC helicoidal e 10 (33,3\%) ressonância magnética abdominal, exames já realizados antes da ecoendoscopia. Dez pacientes (33,3\%) foram submetidos ao USIO. Doze pacientes (40\%) foram submetidos à punção aspirativa com agulha fina (PAAF) na tentativa de confirmar antes da cirurgia a suspeita de insulinoma, evitar falso-positivos ou de realizar o diagnóstico de nódulos linfáticos peri-pancreáticos.

Após a EE, todos (36) foram encaminhados aos seus hospitais de origem para o tratamento. O seguimento dos pacientes e os resultados dos achados cirúrgicos foram obtidos através de telefonemas aos pacientes, familiares, ao médico assistente, quando o mesmo era de outro estado e de consulta ao prontuário médico. 
Excluímos os não operados ou aqueles cujo seguimento foi perdido. Trinta pacientes foram operados (20 mulheres e 10 homens) com média de idade de 44,8 anos (mínima de 14 e máxima de 77 anos).

\section{Equipamentos e técnica}

Os ecoendoscópios utilizados foram: Olympus GIF UM-20 (HIAE) e Pentax FG 32UA e FG 36-UX (UNIFESP) com freqüências de 5, 7,5 e 12,0 MHz. Os exames foram realizados com sedação à base de meperidina, diazepam e propofol em doses individualizadas.

O ecoendoscópio foi introduzido até a segunda porção duodenal. O processo unciforme e a cabeça foram explorados a partir do duodeno e o corpo e a cauda através do estômago. A EE foi bem tolerada por todos os pacientes.

Relatamos os resultados operatórios e do seguimento destes pacientes por um período médio de 3,2 anos (mínimo de quatro meses e máximo de cinco anos). A peça operatória foi examinada, segundo a rotina do serviço de anatomia patológica de cada hospital.

Estudamos os aspectos ecográficos dos tumores quanto à ecogeneicidade (hiperecóica, hipoecóica, isoecóica e anecóica), homogeneidade ou heterogeneidade e se os limites eram nítidos ou não.

\section{Análise estatística}

Os resultados finais foram obtidos após o ato operatório que foi o nosso "gold standard". Avaliou-se a sensibilidade da EE em localizar os tumores e comparou-se com os resultados obtidos pela TC helicoidal, RM e USIO. Utilizou-se o teste exato de Fischer para os cálculos estatísticos e estabeleceu-se como $p<0,05$ os resultados estatisticamente significantes.

\section{Resultados}

Os tumores localizavam-se 12/30 (40\%) no corpo, 9/30 (30\%) na cabeça e 9/30 (30\%) na cauda. $O$ tamanho médio dos insulinomas foi de $1,5 \mathrm{~cm}$ (menor de 0,6 e maior de 5,4 $\mathrm{cm})$. Treze tumores foram menores que $1 \mathrm{~cm}$ (43,3\%), I2 estavam entre $1,1 \mathrm{~cm}$ e $2,0 \mathrm{~cm}$ (40\%) e cinco tinham mais que $2 \mathrm{~cm}(16,7 \%)$ (Figuras I e 2). Apenas um paciente apresentava doença multifocal com três nódulos identificados pela ecoendoscopia e confirmados durante $\mathrm{o}$ ato operatório.

\section{Figura I - Nódulo hipoecóico homogêneo, localizado na cabeça do pâncreas (caso II). 0 US e TC foram negativos para localizar o tumor}

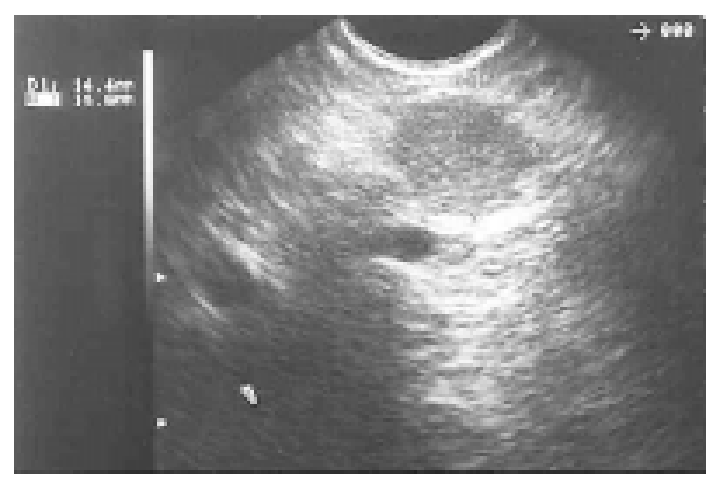

Figura 2 - Nódulo isoecóico, irregular e homogêneo de 2,I x I,6, localizado no corpo do pâncreas

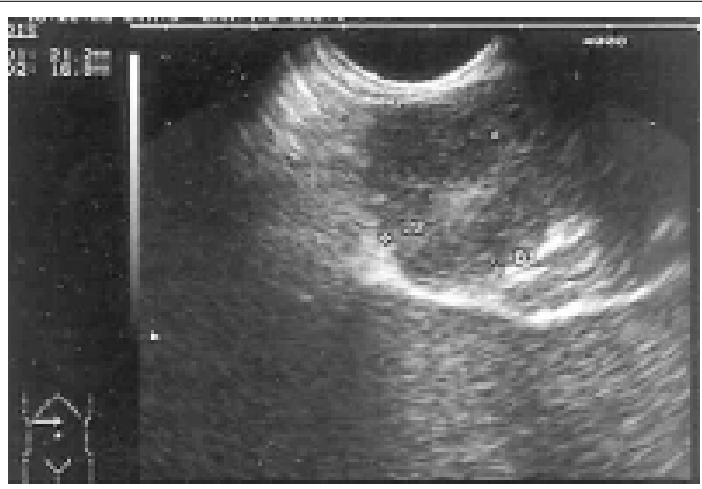

Figura 3 - Punção aspirativa eco-guiada. Citologia revelou a presença de insulinoma

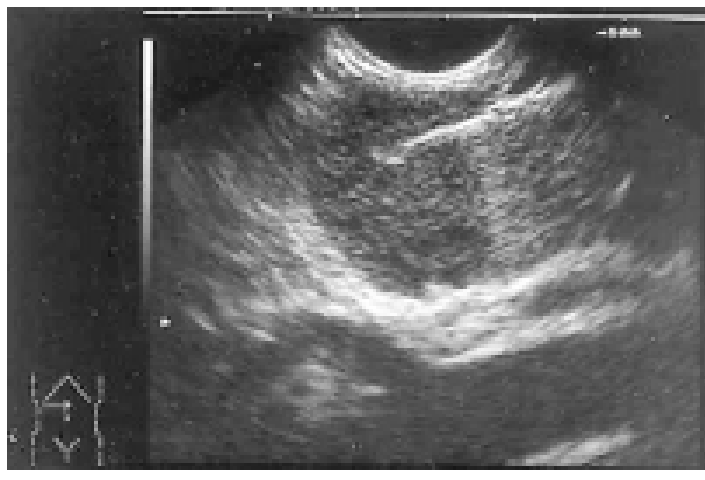

A punção aspirativa com agulha fina ecoguiada (PAAF) para o diagnóstico etiológico pré-operatório foi realizada em 12 pacientes (40\%) (Figura 3). Em dez (83,3\%) foi possível o diagnóstico preciso da lesão, em um caso o resultado foi negativo para a presença de células neoplásicas e em outro não foi possível introduzir a agulha no tumor. O diagnóstico de insulinoma foi feito em cinco casos, de tumor neuroendócrino em cinco. O diagnóstico de insulinoma maligno ocorreu em quatro pacientes (dois diagnosticados pela PAAF e dois pelo histopatológico da peça operatória). 


\begin{tabular}{|c|c|c|c|c|c|c|c|c|c|c|c|}
\hline \multicolumn{12}{|c|}{ Tabela I - Resultados da TC, RM, EE, cirurgia, histologia e seguimento dos 30 pacientes } \\
\hline CASO & ID & $\underline{s}$ & TC/RM/EE & TAMANHO & sítıo & PAAF & CIR. & PALPAÇÃO & USIO & HISTO. & FOLLOW-UP \\
\hline I & $\overline{42}$ & $\mathrm{~F}$ & $+N /-$ & 0,7 & $\overline{\text { CAUDA }}$ & $\mathrm{N}$ & $\overline{P D}$ & - & $\mathrm{N}$ & $B$ & CURA \\
\hline 2 & 68 & $\mathrm{~F}$ & $-\mathrm{N} /-$ & 0,8 & CAUDA & $\mathrm{N}$ & EN & - & t & B & CURA \\
\hline 3* & 49 & $\mathrm{~F}$ & $+\mathrm{N} /+$ & 4,2 & CORPO & TNE & PD & + & N & M & RUIM \\
\hline 4 & 19 & F & $-N /+$ & 2,7 & CABEÇA & $\mathrm{N}$ & GDP & t & $\mathrm{N}$ & B & CURA \\
\hline $5 *$ & 23 & F & $-N /+$ & I & CORPO & NEG & EN & + & N & $B$ & CURA \\
\hline 6 & 30 & $\mathrm{~F}$ & $-\mathrm{N} /+$ & 0,8 & CORPO & $\mathrm{N}$ & EN & + & N & B & CURA \\
\hline 7 & 66 & $\mathrm{~F}$ & $-\mathrm{N} /+$ & 1,4 & CORPO & N & PD & + & N & B & CURA \\
\hline 8 & 33 & $\mathrm{~F}$ & $-\mathrm{N} /+$ & 0,9 & CAUDA & $\mathrm{N}$ & EN & + & N & B & CURA \\
\hline $9 *$ & 36 & F & $-H / t$ & 1,2 & CABECA & TNE & EN & - & t & B & CURA \\
\hline 10 & 46 & $\mathrm{~F}$ & $-N /+$ & 0,8 & CAUDA & $\mathrm{N}$ & EM & t & $\mathrm{N}$ & B & CURA \\
\hline 11 & 47 & M & $-\mathrm{N} /+$ & 1,4 & CABECA & $\mathrm{N}$ & GDP & t & N & M & RUIM \\
\hline 12 & 46 & M & $-N /+$ & 1,9 & CABEÇA & N & EN & + & N & B & CURA \\
\hline $13 *$ & 51 & $\mathrm{~F}$ & $-\mathrm{N} /+$ & 0,8 & CAUDA & TNE & EN & - & t & M & RUIM \\
\hline 14 & 54 & $\mathrm{~F}$ & $-\mathrm{N} /+$ & 1,2 & CABEÇA & $\mathrm{N}$ & EN & + & N & B & CURA \\
\hline 15 & 41 & M & $-\mathrm{N} /+$ & 2 & CABEÇA & N & GDP & + & N & B & CURA \\
\hline 16 & 38 & $\mathrm{~F}$ & $+\mathrm{N} /$ & 1,8 & CAUDA & N & PD & - & - & $B$ & CURA \\
\hline 17 & 44 & F & $-\mathrm{N} /+$ & | & CABECA & N & EN & t & N & B & CURA \\
\hline 18 & 37 & F & $-1+1-$ & 1,5 & CAUDA & $\mathrm{N}$ & EN & t & t & $B$ & CURA \\
\hline 19 & 33 & $M$ & $+\mathrm{N} /+$ & 2 & CAUDA & $N$ & PD & - & + & B & CURA \\
\hline 20 & 51 & M & $-N /+$ & 0,8 & CORPO & $\mathrm{N}$ & EN & + & $\mathrm{N}$ & B & CURA \\
\hline $21 *$ & 55 & $\mathrm{~F}$ & $-H+$ & | & CABECA & TNE & EN & t & N & B & CURA \\
\hline 22 & 77 & $\mathrm{~F}$ & $+\mathrm{N} /+$ & 1,4 & CORPO & $N$ & EN & - & + & B & CURA \\
\hline $23 *$ & 36 & $\mathrm{~F}$ & $-/-1+$ & 1,6 & CORPO & $\mathbb{N}$ & $\mathrm{EN}$ & - & t & B & CURA \\
\hline 24 & 57 & M & $+\mathrm{N} /+$ & 0,6 & CAUDA & $\mathrm{N}$ & EN & - & t & $B$ & CURA \\
\hline $25 *$ & 48 & M & $+/ t / t$ & 2,4 & CABECA & $\mathbb{N}$ & GDP & + & $\mathrm{N}$ & B & CURA \\
\hline $26 *$ & 14 & M & $+/ t / t$ & 2,2 & CORPO & $\mathbb{N}$ & EN & + & $\mathrm{N}$ & B & CURA \\
\hline $27 *$ & 62 & $\mathrm{~F}$ & $-1-1+$ & 0,7 & CORPO & INS & EN & + & t & B & CURA \\
\hline 28* & 58 & M & $+1-1+$ & 1,6 & CORPO & TNE & PD & + & N & $B$ & CURA \\
\hline $29 *$ & 49 & $\mathrm{~F}$ & $+1+1+$ & 5,4 & CORPO & $\mathbb{N}$ & PD & t & N & M & RUIM \\
\hline 30* \# & 36 & $M$ & $-\mathrm{N} /+$ & 0,8 & CORPO & $\mathbb{N}$ & PD & + & + & B & CURA \\
\hline
\end{tabular}

$(I D=$ Idade, $S=$ Sexo, F = Sexo feminino, M = Sexo masculino, TC = Tomografia computadorizada, TNE = Tumor neuroendócrino, $+=$ Lesão presente ou palpável. - Lesão ausente aos exames a palpação, RM = Ressonância magnética, EE = Ecoendoscopia, CIR. = Cirurgia, PD = Pancreatectomia caudal, EN = Enucleação, GDP = Gastroduodeno-pancreatectomia, USIO = Ultra-som intra-operatório, N = Não foi realizada, HISTO. = Histologia, $\mathrm{B}=$ Benigno e $\mathrm{M}=$ Maligno).

* = Pacientes submetidos à PAAF.

\# = Paciente com doença multifocal.

O US convencional identificou o insulinoma em I/30 (3,3\%). A sensibilidade da EE $(86,6 \%)$ foi superior à do US $(3,3 \%, p<0,05)$. A TC helicoidal identificou $33,3 \%$ e a EE $86,6 \%$ dos tumores o teste exato de Fischer também mostrou significância estatística $(p<0,05)$. A ressonância magnética foi realizada em dez pacientes antes da EE e reparou o tumor em quatro (40\%), três confirmados pela EE e um confirmado pelo USIO e enucleação $(p<0,05)$.

Onze pacientes (36,6\%) foram submetidos ao USIO. Em I0/II (91\%) esse exame identificou a lesão, ao compararmos com a $E E$, não houve significância estatística ( $p>0,05)$. O USIO falhou em um doente que apresentava lesão na cauda, que já tinha o diagnóstico confirmado pela
TC helicoidal. Em um doente apenas esse exame conseguiu identificar o tumor com precisão.

A TC foi o único método a identificar o tumor em 2/30 pacientes. Em I 5 pacientes a $E E$ foi o único método que identificou a lesão (50\%). O USIO apresentou sensibilidade de $90,9 \%$, pouco superior a taxa da EE que foi de $86,6 \%$. Nas lesões localizadas na cauda do pâncreas a TC, EE, RM, e USIO apresentaram sensibilidades de $44,4 \%$, $55,5 \%, 50 \%$ e $83,3 \%$, respectivamente. A EE identificou todos os tumores localizados na porção cefálica e corpórea do pâncreas. A sensibilidade global e por região do pâncreas de cada um dos métodos utilizados nesse estudo podem ser apreciados na Tabela 2.
Dos tumores identificados pela EE 24/26 (92,3\%) apresentavam limites nítidos. Os dois malignos de maior tamanho tinham calcificações centrais, eram irregulares, heterogêneos, hiperecóicos e de limites imprecisos. Dos 22 benignos identificados pela EE, 14 eram hipoecóicos $(63,6 \%)$, oito isoecóicos (36,4\%) e todos (100\%) homogêneos e regulares (Figuras | e 2).

Dezoito pacientes (60\%) foram submetidos à enucleação, oito à pancreatectomia distal $(26,6 \%)$ e quatro à gastroduodenopancreatectomia (13,4\%). Os pacientes com tumores malignos faleceram cinco e seis meses após a cirurgia. Os outros foram seguidos ambulatorialmente e os resultados desse seguimento podem ser vistos na Tabela I. 
Ardengh JC et al.

Tabela 2 - Acurácia dos vários testes utilizados na localização pré-operatória da lesão primária na glândula pancreática, segundo o local onde foram encontrados os tumores pelo cirurgião

\begin{tabular}{|c|c|c|c|c|c|}
\hline Local & TC (\%) & RM (\%) & $\mathrm{EE}(\%)$ & Palpação (\%) & USIO (\%) \\
\hline $\begin{array}{l}\text { Cabeça } \\
\text { Corpo } \\
\text { Cauda }\end{array}$ & $\begin{array}{l}1 / 9(I 1, I) \\
5 / 12(41,6) \\
4 / 9(44,4)\end{array}$ & $\begin{array}{l}\mid / / 3(33,3) \\
2 / 5(40) \\
1 / 2(50)\end{array}$ & $\begin{array}{c}9 / 9(100) \\
12 / 12(100) \\
5 / 9(55,5)\end{array}$ & $\begin{array}{c}8 / 9(88,8) \\
10 / 12(83,3) \\
3 / 9(33,3)\end{array}$ & $\begin{array}{l}1 / 1(100) \\
4 / 4(100) \\
5 / 6(83,3)\end{array}$ \\
\hline ACURÁCIA & $10 / 30(33,3)$ & 4/10 (40) & $26 / 30(86,6)$ & $21 / 30(70)$ & $10 / 1 \mid(90.9)$ \\
\hline
\end{tabular}

US = Ultra-som abdominal, TC = Tomografia computadorizada, EE = Ecoendoscopia, RM = Ressonância magnética, USIO = Ultra-som intra-operatório.

\section{Discussão}

A palpação intra-operatória é o método mais sensível para localizar os tumores endócrinos do pâncreas com sensibilidade ao redor de $90 \%{ }^{8}$. A localização exata pré-operatória desse tipo de tumor, o diagnóstico de malignidade e a identificcação de metástases hepáticas tem por objetivo a programação adequada do tratamento cirúrgico, reduzindo a morbi-mortalidade e favorecendo a evolução no pós-operatório ${ }^{1,9}$.

Neste estudo, a acurácia na localização dos tumores pelo US foi de 3,4\% enquanto a TC foi de 33,3\%. O US apresenta acurácia que varia de $0 \%$ a $62 \%$ e a acurácia da TC helicoidal varia de $12 \%$ a $73 \% \%^{5,10-14}$. Os resultados preliminares envolvendo a ressonância magnética do pâncreas na identificação dessas lesões são desapontadores ${ }^{15,16}$, o que foi confirmado nos 10 pacientes submetidos a esse teste antes da realização da EE, que apresentou acurácia de $40 \%$. Métodos invasivos como a angiografia (AG) e CVTH têm melhores resultados. A sensibilidade da $A G$ varia de $44 \%$ a $67 \% 5,17,18,19$, porém em outros o índice de acerto foi de $33 \%^{16,20,21}$. A CVTH tem acurácia de $64 \%$ a 100\%, entretanto é demorada, necessita de um profissional experiente e apresenta várias complicações como: epigastralgia, hemobilia transitória, hematoma hepático, extravasamento biliar no peritônio e hemorragia $3,13,16,21$.

O USIO combinado à palpação apresenta sensibilidade de $90 \%$ a $100 \%$. Entretanto, esse método prolonga a cirurgia, além de apresentar o risco de lesão dos vasos esplênicos devido a mobilização da glândula pancreática do retroperitônio. Mesmo assim, o diagnóstico preciso é em muitos casos difícil de ser obtid0 ${ }^{5,22}$. Esses achados corroboram com os resultados desse estudo que demonstraram acurácia de 90,9\%.

A identificação precisa do local do tumor no pré-operatório evitaria o risco imposto pela técnica do USIO associado à palpação, possibilitando uma ressecção operatória planejada, principalmente na suspeita de malignidade ${ }^{5,22}$. 0 estudo das estruturas vizinhas ao tumor (ducto pancreático principal, artéria e veia esplênica, veia porta e veia mesentérica superior) é fundamental para o planejamento da estratégia operatória a ser adotada; enucleação do tumor ou ressecção pancreática ${ }^{23}$.

A EE tem sensibilidade semelhante à CVTH e ao USIO na detecção de tumores pequenos do pâncreas ${ }^{1,3}$. Comparado a outros estudos prospectivos, a taxa de sensibilidade encontrada por Glover et al. ${ }^{6}$ em 14 pacientes foi de $79 \%$, Zimmer et al..$^{24} \mathrm{em}$ seis pacientes apresentaram taxa de $90 \%$ e Ardengh et al. ${ }^{24} \mathrm{em} 12$ pacientes apresentaram taxa de $83,3 \%$. Na casuística atual foi possível determinar o local preciso em $86,6 \%$ dos pacientes.

Schumacher et al..$^{2}$, em estudo prospectivo com a EE em 14 pacientes, encontraram baixa sensibilidade no diagnóstico (57\%). Isso ocorreu pois a maioria dos tumores estudados localizavam-se na cauda. Esse autor localizou $3 / 8$ tumores da cauda (37,5\%) e 5/6 da cabeça (83\%). Esses resultados são semelhantes aos nossos, quando analisamos os resultados obtidos com a EE no diagnóstico dos insulinomas localizados na porção caudal do pâncreas, onde obtivemos sensibilidade de 55,5\%. Na porção cefálica e corpórea nossos resultados são superiores aos relatados por esse autor ( $100 \%$ vs $83 \%$ ).

Em nossa casuística a maioria dos pacientes apresentavam lesões no corpo do pâncreas, o que corrobora com os achados de outros estudos da literatura onde a maioria dos tumores estudados se encontram na cabeça e corpo ${ }^{4,6}$. Zimmer et al. ${ }^{25}$, em seis pacientes onde cinco deles tinham nódulos na cauda, apresentaram taxa de sensibilidade superior, mas isso deve-se ao fato de que a maioria dos pacientes apresentarem lesões múltiplas o que facilitou a identificação dos mesmos.
A falha da EE ocorre em tumores menores que I cm, localizados no corpo ou cauda do pâncreas, ou em lesões pedunculadas². Em nossa série, 4/9 pacientes, com tumores menores que $2 \mathrm{~cm}$, localizados na cauda não foram vistos pela $\mathrm{EE}(44,4 \%)$.

A PAAF pode se tornar o método de escolha nesta doença, pois confirma pelo exame citológico o diagnóstico clínico de insulinoma diferençando a doença maligna da benigna e orientando a melhor forma de abordagem cirúrgica desses casos. Corroborando com esta acertiva, Santo \& Giovannini ${ }^{26}$ estudaram 26 pacientes com síndrome neuroendócrina e concluíram que a EE associada à PAAF é efetiva no diagnóstico etiológico e identificação desses tumores, devendo ser realizada como exame de primeira escolha nesses pacientes. Em nossa casuística, 12 pacientes foram submetidos à PAAF na tentativa de elucidar o diagnóstico histológico no pré-operatório, o que foi conseguido em 10/12 pacientes $(83,3 \%)$, resultado inferior se comparado com os encontrados em outra série que foi de $92 \%{ }^{26}$. Essa técnica é interessante nos pacientes com suspeita de tumores neuroendócrinos do pâncreas, visto que sua indicação estaria baseada na tentativa de confirmar com exatidão a presença da lesão, evitando assim um possível resultado falso-positivo. Outro fator de fundamental importância para sua realização seria a possibilidade de diferençar uma lesão maligna de uma benigna e de fazer a distinção entre um nódulo linfático peri-pancreático de um tumor neuroendócrino ${ }^{27}$.

\section{Conclusão}

Os resultados obtidos através da análise da presente casuística permitem concluir que, apesar de invasiva, a EE em mãos experientes é um método eficiente para a localização dos insulinomas antes da 
cirurgia, sua sensibilidade global é superior à da TC helicoidal e ressonância magnética abdominal. A PAAF é um método complementar que pode fazer o diagnóstico de certeza do pequeno nódulo encontrado, diminuindo as chances de um resultado falso-positivo.

\section{Conflito de interesse: não há.}

\section{SUMMARY}

\section{IDENTIFICATION OF INSULINOMAS BY ENDOSCOPIC ULTRASONOGRAPHY}

BACKGROUND. The aim of this study is to compare EUS and the others diagnostics tests in the correct localization of insulinomas.

METHODS. We prospectively investigated 30 patients with endoscopic ultrasound with a clinical diagnosis of insulinomas prior to surgical exploration. They were submitted to abdominal ultrasonography, spiral computed tomography and four patients were submitted to magnetic ressonance before EUS. Surgery was the gold standard for tumor localization.

RESULTS. Twenty-six tumors were benign (86.6\%) and four were malign (13.4\%). The median size tumors detected by EUS was $1.5 \mathrm{~cm}$. The overall sensitivity of EUS in identifying insulinomas was $86.6 \%$ compared to $33 \%$ for CT, $40 \%$ to MRI and $90.9 \%$ to IUS. In 12 patients we were able to perform EUS-guided fine needle aspiration. Insulinoma was diagnosed in ten cytological specimens (83.3\%). Tumors located in the head and body of the pancreas were seen by EUS in all patients, respectively but those located in the tail were diagnosed only in $55.5 \%$ of the cases.

Conclusions. EUShas a high sensibility in the identification and localization of pancreatic insulinomas and should replace traditional methods of image when clinical suspicion is high. [Rev Assoc Med Bras 2004; 50(I): |67-7I]

KEY WORDS: Diagnosis. Insulinomes. Endoscopic Ultrasound. Fine needle aspiration.

\section{REFERÊNCIAS}

I. Pitre J, Soubrane L, Chapuis, Y. Endoscopic ultrasonography for insulinomas. Pancreas 1990; 13:55-60

2. Shumacher B, Lubke HJ, Frieling T. Prospective study on the detection of insulinomas by endoscopic ultrasonography. Endoscopy 1996; 2: $273-6$.

3. King CMP, Reznek RH, Dacie JE, Waas JA. Review imaging islet cell tumors. Clin Radiol 1994: 49:295-303.

4. Rösch T, Lightdate CJ, Bolet JF, Boyce GA, Sivak MV, Yasuda K, et al. Localization of pancreatic endocrine tumor by endoscopic ultrasonography. N Engl J Med 1992; 326: I 721-6.

5. Galiber AK, Reading CC, Charboneau JW, Sheedy PF, James EM, Gorman, B, et al. Localization of pancreactic insulinoma. Comparison of pre and intraoperative US with CT and angiography. Radiology 1988; 166:405-8.

6. Glover Jr, Shorvon PJ, Lees WR. Endoscopic ultrasound for localization of islet cell tumors. Gut 1992; 33:108-10.

7. Thompson NW, Czako PF, Fritts LL, Bude R, Bensol R, Nostrant TT, et al. Role of endoscopic ultrasonography in the localization of insulinomas and gastronomas. Surgery 1994; I | 6: | | 3 |-8.

8. Daggett PR, Goodburn EA, Kurtz AB, Le Quesne LP, Morris DV, Nabarro JD, et al. Is preoperative localization of insulinomas necessary? Lancet 1981; I (82 I 8):483-6.

9. Fedorak II, Ko TC, Gordon D, Flisak M, Prinz RA. Localization of islet cell tumors of the pancreas: a review of current techniques. Surgery 1993; 1 13:242-9.

I0. Krudy A, Doppman JL, Jensen RT, Norton JA, Collen MJ, Shawker TH, et al. Localization of islet cell tumors by dinamic CT: Comparison with plain $\mathrm{CT}$, arteriography, sonography and venous sampling. AJR 1994; I43:585-9.

II. Rossi P, Allison DJ, Bezzi M, Kennedy A, Maccioni F, Winick D, et al. Endocrine tumors of the pancreas. Radiol Clin North Am 1989; 27:|29-6I.

12. Meyenberger C, Bertschinger P, Zala GF, Marincek B. Endosonography in diagnosis of insulinoma. Ultraschall Med 1995; 16:224-7.

13. Kisker O, Bastian D, Frank M, Rothmund M. Diagnostic localization of insulinoma. Experience with 25 patientes with solitary tumors. Med Klin 1996; 91:349-54.

14. Kuzin NM, Egorov AV, Konarashin SA, Lotova N, Kuznetzov NS, Majarovi JB. Pre-operative and intraoperative topographic diagnosis of insulinomas. World J Surg 1998; 22:593-7.

I5. Broughan TA, Leslie JD, Soto JM, Hermann RE. Pancreatic islet cell tumors. Surgery 1986; 99:67।-8.

16. Vinik Al, Delbridge L, Moattari R, Cho K, Thompson N. Transhepatic portal vein cathe- rization for localization of insulinomas: a ten year experience. Surgery 1991; I09: I-I I.

17. Proye C, Boissel P. Preoperative imaging versus intraoperative localization of tumors in adult surgical patientes with hiperinsulinemia: A multicenter study of 338 patients. World J Surg 1988; 12:685-90.

18. Rothmund M, Angelini L, Brunt LM, Farndon JR, Geelhoed G, Grama D, et al. Surgery for benign insulinoma: an international review. World J Surg 1990; 14:393-9.

19. Gianello P, Gigot JF, Berthet F, Dardenne NA, Lambotte L, Raheer J, et al. Pre and intraoperative localization of insulinomas: report of 22 obervations. World J Surg 1988; 12:389-97

20. Doherty GM, Doppman JL, Shawker TH, Miller DL, Eastman RC, Gorden P, et al. Results of a prospective strategy to diagnose, localize and resect insulinomas. Surgery 1991; I I 0:989-97.

21. Roche A, Raisonnier A, Gillon-Savouret MC. Pancreatic venous sampling and arteriography in localizing insulinomas and gastrinomas: procedures and results in 55 cases. Radiology 1982; | 45:62।-7.

22. Norton JA, Cromack DT, Shawher TH, Doppman JL, Comi R, Garden P, et al. Intraoperative ultrasonographic localization of islet cell tumors - A prospective comparison to palpation. Ann Surg 1988; 207: 160-8.

23. Stipa V, Chirletti P, Coronna R. Diagnostic and therapeutic strategy of insulinomas. A propos of a personal experience of 21 cases. Chirurgie 1997; 121:667-7|

24. Ardengh JC, Rosenbaum P, Ganc AJ, Goldenberg A, Lobo EJ, Malheiros CA, et al. Role of EUS in the preoperative localization of insulinomas compare with spiral CT. Gastrointest Endosc 2000; 51:552-5.

25. Zimmer T, Stölzel U, Bäder M, Koppenhagen K, Hamm B, Buhr H, et al. Endoscopic ultrasonography and somatostatin receptor scintigraphy in the preoperative localization of insulinomas and gastrinomas. Gut 1996; 39:562-8.

26. Santo E, Giovannini M. Role of endoscopic ultrasonography (EUS) and EUS guided fine needle aspiration in the evaluation of neuroendocrine tumors of the pancreas [abstract]. Digestion 1998; 5:42.

27. Ginès $A$, Vazquez-Sequeiros E, Soria MT, Clain JE, Wiersema MJ. Usefulness of EUSguided fine needle aspiration (EUS-FNA) in the diagnosis of functioning neuroendocrine tumors. Gastrointest Endosc 2002; $56: 291-6$.

Artigo recebido: I //04/03 Aceito para publicação: 01/09/03 